\title{
ESTILOY DECORACIÓN: METODOLOGÍA PARA EL RELEVAMIENTO DE VASIJAS CERÁMICAS DE LA QUEBRADA DE HUMAHUACA (PROVINCIA DE JUJUY, ARGENTINA)
}

\author{
MARÍA ANDREA RUNCIO \\ UNIVERSIDAD DE BUENOS AIRES \\ andrearuncio@hotmail.com
}

\section{RESUMEN}

El trabajo presenta la metodología desarrollada para el relevamiento de la decoración de un conjunto de vasijas provenientes de la Quebrada de Humahuaca (Provincia de Jujuy, Argentina) en base al análisis de variables independientes y su posterior correlación que permitió dar cuenta de la variabilidad inherente en todo conjunto cerámico.

Palabras ClaVes: metodología, decoración, vasijas cerámicas.

\section{ABSTRACT}

In this paper the methodology that has been developed for the record of the ceramic decoration from the Quebrada de Humahuaca (Provincia de Jujuy, Argentina) is presented. It is based on the analysis of independent variables and its subsequent correlation, which allowed recognizing the variability inherent in all ceramic assemblage.

KEYWORDS: methodology, decoration, ceramic vessels.

\section{INTRODUCCIÓN}

La cuestión estilística en el campo de la arqueología es fundamental en el análisis e interpretación del registro arqueológico. La definición de estilos se vincula al problema de la variabilidad en la cultura material, siendo los estilos los que permiten ordenar esa variabilidad. Es decir, a partir de similitudes y diferencias en la cultura material se conforman conjuntos de objetos semejantes que conforman los estilos.

Partiendo de esta concepción amplia de estilo, se desarrollaron a lo largo de la historia de la arqueología una serie de enfoques que enfatizan diferentes aspectos de la cuestión estilística (Longacre 
1968; Sackett 1977; Wobst 1977; Wiessner 1983; Hodder 1985; Shanks y Tilley 1992, entre otros) aunque -como sostiene Rice (1987: 245)- los arqueólogos a menudo han evitado dar definiciones explícitas de "estilo".

De acuerdo con esta autora, los arqueólogos frecuentemente utilizan el término estilo principalmente para referirse al estilo decorativo, es decir, el embellecimiento de la superficie de un objeto (Rice 1987: 245), desarrollándose diferentes propuestas metodológicas para el relevamiento de este estilo decorativo o decoración, especialmente en vasijas cerámicas.

En este trabajo utilizaré el término estilo para referirme, en sentido amplio, a un conjunto de expresiones materiales correspondientes a un contexto espacio-temporal particular donde se plasman los significados culturales propios de la sociedad que las produce y, por lo tanto, la identifica.

Siguiendo la propuesta de Rocchietti $(1991,1995,2002,2008)$ el estilo se conforma en la tensión entre coherencia y variación. Coherencia en cuanto a similitudes que responden a una regla de realización producto de una cultura compartida y variación que se verifica en signos únicos o combinaciones únicas de los mismos. El estilo se constituye, así, en una asociación histórica específica derivada de la sujeción a una norma convencional y a ejercicios de su trasgresión que permiten evaluar el peso del código cultural bajo el cual se elaboraron (1991: 25).

Con el término decoración se alude específicamente a las representaciones y manifestaciones plásticas que aparecen en las superficies de las expresiones materiales que conforman el estilo. Por lo tanto, estilo y decoración están indisolublemente asociados.

Presentaré, en primer término, una revisión de las diferentes propuestas de registro de la decoración cerámica; posteriormente, describiré la metodología desarrollada para el relevamiento de la decoración de un conjunto de vasijas provenientes de la Quebrada de Humahuaca (Provincia de Jujuy, Argentina) y finalmente, ejemplificaré su aplicación con el caso de las ollas subglobulares.

\section{DIFERENTES MÉTODOS DE RELEVAMIENTO DE LA DECORACIÓN CERÁMICA}

Una amplia variedad de términos han sido utilizados para describir los componentes específicos o unidades analíticas de la decoración.

El enfoque tradicional de análisis de la decoración cerámica, se centra en la identificación de componentes del diseño decorativo y el agrupamiento de las vasijas o los tiestos en tipos o clases (estilos) en base a la presencia o ausencia de esos componentes.

Hay cuatro componentes principales (elemento, motivo, configuración y estructura) que, siguiendo a Rice (1987: 248-9) pueden definirse de la siguiente forma:

Elemento: componente independiente más pequeño de un diseño que puede ser manipulado como una unidad simple.

Motivo: combinaciones de elementos que son usadas para formar componentes más amplios de la decoración. Usualmente son lo suficientemente amplios o complejos como para llenar la mayor parte del espacio decorativo y generalmente aparecen en grupos más que individualmente.

Configuración: es la forma en que los motivos son dispuestos para llenar una división espacial, constituyendo un complejo visual que se reconoce como "diseño decorativo". 
Estructura: se refiere al lugar donde aparece la decoración en la superficie de la vasija, si está subdividida y de qué forma, la simetría y el balance de la decoración, la cantidad de espacio cubierto y el lugar y relación de los diferentes elementos, motivos y configuraciones.

A partir de la década del 60, surgieron diferentes métodos de relevamiento de la decoración que, de acuerdo con Rice (1987: 252-266), pueden sintetizarse en tres grandes grupos:

\section{1) Análisis de los elementos de diseño}

El análisis de los elementos de diseño procura aislar los elementos individuales del diseño correspondientes a un estilo particular y explicar su distribución espacial en términos del comportamiento social de los que producen ese estilo. Específicamente, este enfoque ha sido utilizado para medir la similitud de elementos de diseño de un estilo particular entre grupos sociales diferentes, dentro de un grupo particular y entre distintos estilos de un mismo grupo o de grupos diferentes. El procedimiento implica, en primer lugar, la recopilación de todos los elementos de diseño que aparecen en los estilos de interés; luego se tabula la frecuencia de aparición de cada elemento asociada con cada grupo o subgrupo y por último, estas frecuencias pueden compararse directamente o a través de algún test estadístico para expresar la similitud entre estilos (Rice 1987: 252).

Este enfoque ha recibido tres críticas principales.

La primera se refiere a la definición de los elementos de diseño. En la mayoría de los análisis el criterio ha sido impreciso y subjetivo y los procedimientos usados en la definición y medida de los elementos directamente no son explicitados (Rice 1987: 257).

La segunda crítica se refiere al tamaño de las muestras analizadas. Por un lado, cuando se trabaja con tiestos, es necesario calcular algún tipo de estimación de Número Mínimo de Vasijas ya que, de lo contrario, puede aumentar el número de elementos tabulados. Por el otro, algunos autores sugieren que si se trabaja con muestras menores a 75-100 casos por locus de interés (sitio, recinto), los cálculos estadísticos de similitud o correlación no son confiables (Rice 1987: 257-8).

La última crítica se relaciona con el uso inadecuado de modelos estadísticos para calcular la similitud entre unidades de procedencia (Rice 1987: 258).

Estas críticas al análisis de elementos de diseño condujeron a nuevas formas de medición de la variabilidad entre elementos. Así, se ha propuesto el uso de atributos y estados de atributo. Los atributos son características medibles de los elementos o motivos con estados alternativos claros (estados de atributo). Además, puede medirse la variación temporal y espacial en un atributo, viendo la frecuencia de diferentes estados de atributo a través del tiempo y del espacio (Rice 1987: 259).

\section{2) Análisis de simetría}

Este enfoque usa un conjunto de términos para describir la propiedad de simetría en relación a la posición espacial de figuras geométricas y su movimiento a lo largo de una línea o alrededor de un punto. El análisis de simetría de la decoración cerámica implica la identificación y descripción de un patrón de diseño. El proceso comienza identificando la unidad básica o parte fundamental del patrón de diseño, es decir, la parte o unidad a partir de la cual puede generarse la composición total, que puede ser tanto un elemento como un motivo en la terminología convencional. Luego se determina el movimiento por el cual esa parte es repetida en la vasija o la transformación por la cual es movida 
alrededor de un punto o línea real o imaginario para generar el patrón de diseño. Hay cuatro clases de movimientos y, por lo tanto, de simetrías: traslación, simetría bilateral o reflexión especular, rotación y reflexión traslatoria. Si el diseño se compone de una única parte fundamental que no se repite, se denomina asimétrico (Rice 1987: 260-1).

\section{3) Análisis de la estructura del diseño}

Este enfoque postula una organización jerárquica en la ejecución del diseño donde el nivel más alto de esa jerarquía es el sistema de división espacial de la vasija. Los diseños que se ubican en esas divisiones espaciales tienen dos niveles de organización: elementos y configuraciones de diseño. Los elementos se definen como la unidad independiente más pequeña y las configuraciones como los arreglos de elementos de diseño que tienen la suficiente complejidad como para llenar una división espacial. Los elementos primarios de diseño se pintan primero mientras que los elementos secundarios son opcionales y cuando se los agrega, su ubicación en la configuración depende de la clase de elemento primario usado (Rice 1987: 264).

Pueden mencionarse tres ventajas respecto de la utilización del enfoque jerárquico antes mencionado (Douglass y Lindauer 1988). En primer término, este modelo permite, gracias al arreglo jerárquico de las unidades de diseño, la evaluación de la producción del diseño la cual involucra reunir partes separadas en un todo a través de un proceso paso a paso, donde las primeras construcciones condicionan las posteriores. La segunda ventaja se relaciona con su posibilidad de aplicación a fragmentos. El sistema jerárquico permite descomponer el diseño en distintos niveles de unidades. Estos diferentes niveles pueden ser identificados de acuerdo a la proporción de diseño preservado en el tiesto. Finalmente, la tercera ventaja se relaciona con la posibilidad de establecer divisiones útiles para distinguir entre unidades o estados de atributo cuyas funciones dentro de un diseño no son intercambiables.

Sin embargo, este enfoque también ha recibido críticas (Jerningan 1986). Entre ellas puede mencionarse que si bien el modelo jerárquico se presenta como aplicable universalmente, en muchos casos los elementos no pueden distinguirse de los motivos y los motivos de las estructuras, por lo tanto falla en proporcionar un marco de referencia para comparar amplias tradiciones estilísticas. También se ha criticado que las definiciones de los niveles jerárquicos son ambiguas, conduciendo a confusiones cuando se trata de aplicarlo.

Jerningan (1986) propone un análisis no jerárquico del diseño donde se definen los componentes del diseño usando un único nivel. Esto se logra determinado la manera en cómo los componentes del diseño son usados en el corpus de diseños de un estilo particular. La clave de este enfoque está en aislar unidades de diseño que el autor denomina schema. Este se define como una configuración o patrón de configuraciones que fue concebida como una unidad distintiva por los ejecutores del estilo (1986: 9). La evidencia para la determinación de un schema consiste en la manera en la cual la configuración o el patrón son usados a través de un número de vasijas o contextos de diseño. Entonces, siguiendo al autor un schema consiste en "a complex unit of design consisting of a configuration or pattern of configurations that retains its distinct identity across a number of vessels and/or design contexts in a particular style repertory. A ceramic style is the repertory of schema used by an individual, a social group, a community, a network of communities, a culture or a group of related cultures" (1986: 10-11).

Este enfoque no asume la existencia de niveles jerárquicos sino sólo la distinción de schemata todos en un mismo nivel. Los schemata abarcan no sólo los diseños sino también el arreglo espacial de los mismos en la vasija los cuales son considerados y listados de la misma forma que los diseños. 


\section{Metodología PARA EL RELEVAMIENTO DE LA DECORACIÓN DE VASIJAS CERÁMICAS DE LA QuEBRAdA DE HuMAHUACA}

La Quebrada de Humahuaca está ubicada en la provincia de Jujuy, en el noroeste Argentina. La ocupación prehistórica de esta región ha sido dividida en cuatro períodos: Temprano (300-700 d.C.), Medio (700-900 d.c.), Tardío (900-1480 d.c.) e Inca (1480-1536 d.C.).

La cerámica de producción local se caracteriza por un conjunto de formas abiertas y cerradas que corresponden a pucos, ollas, cántaros, jarras, vasos chatos, entre otras, de color rojo con decoración geométrica pintada en negro y minoritariamente en blanco y negro que, en general, se presenta sobre superficies pulidas. Los diseños característicos comprenden líneas, reticulados, triángulos, espirales, entre otros, que se combinan formando diferentes motivos. Estos aparecen tanto en las superficies internas de pucos y otras formas abiertas como en el exterior de ollas y cántaros. Se presentan, asimismo, pucos con la superficie interna pulida de color negro, ollas que presentan filas de incisiones en sus cuellos y un conjunto de vasijas ordinarias, sin decoración y con sus superficies alisadas.

Estas vasijas forman parte de un estilo regional utilizado en la Quebrada de Humahuaca durante los períodos Tardío e Inca.

Como parte de mi Tesis Doctoral (Runcio 2010) estudié un conjunto de 1719 vasijas correspondientes a los periodos antes mencionados. Las mismas forman parte de las colecciones de varios museos: Museo Etnográfico "Juan B. Ambrosetti” y Museo Arqueológico "Eduardo Casanova" (ambos dependientes de la Facultad de Filosofía y Letras de la Universidad de Buenos Aires), Museo Arqueológico e Histórico de Huacalera (Provincia de Jujuy) y Museo de Ciencias Naturales (Universidad Nacional de La Plata).

Presentaré a continuación la metodología desarrollada para el relevamiento de la decoración de estas piezas, ejemplificándola en este caso con el análisis de las ollas subglobulares.

La concepción del estilo ya mencionada como el interjuego entre la coherencia y la variación (Rocchietti 1991, 1995, 2002, 2008) implica adoptar un método de análisis que pueda dar cuenta de ambas categorías.

Además, varios trabajos críticos sobre los estilos cerámicos establecidos para la Quebrada de Humahuaca plantean la dificultad para adscribir las vasijas a alguno de los estilos propuestos, dado que la variabilidad en la combinación de formas y decoración es mucho mayor que la contemplada en la definición de los mismos (Palma 1996; Nielsen 1997; Rivolta 1997).

Por lo anteriormente mencionado, se decidió desagregar el análisis de la decoración en diferentes variables. En un primer momento, se consideró cada una de manera independiente evaluando, posteriormente, la interrelación entre las mismas. El análisis independiente de cada variable y su posterior correlación permitió reconocer agrupaciones significativas contemplando múltiples posibilidades de asociación y similitud. A partir de este procedimiento, entonces, fue posible sistematizar los datos para generar tendencias más finas, evitando reducir a priori la variabilidad existente y no constriñéndola a los estilos ya establecidos.

Las variables de análisis consideradas fueron tres:

1. Forma de la pieza.

2. Tratamiento de superficie.

3. Diseño decorativo, donde se discriminó la(s) unidad(es) de diseño presente, el arreglo espacial y la estructuración de las mismas en la cara interna y/o externa de la pieza. 


\section{Forma de la pieza}

Las formas corresponden a los distintos tipos de piezas abiertas y cerradas tanto locales como incas o con influencias incaicas que han sido definidas para la Quebrada de Humahuaca. Se han considerado 8 Grupos Morfológicos (Pucos, Ollas, Cántaros, Jarras, Vasos chatos, Pelikes, Aribaliformes y Platos) divididos en diferentes subgrupos siguiendo la clasificación de Palma (1998) (Fig. 1).

Las ollas subglobulares se caracterizan porque el cuerpo tiene -desde la base- paredes marcadamente convexas. El cuello es estrecho y poseen borde evertido. Presentan asas verticales que unen el borde o el cuello con el tercio superior del cuerpo u horizontales ubicadas en el medio del cuerpo, a la altura del diámetro máximo. La base puede ser plana o convexa (Figs. 1 y 2).

\section{Tratamiento de superficie}

Para el tratamiento de superficie se consideraron dos categorías: alisado y pintado-pulido, que constituyen las principales formas de acabado de superficie de las cerámicas de la Quebrada de Humahuaca (Palma 1996; López 2000-2002, 2004).

Con respecto al tratamiento pintado-pulido se han distinguido siete variantes para las piezas locales: rojo, negro/rojo, marrón y gris que se presentan mayoritariamente y en menor medida, blanco y negro/sobre rojo, blanco/rojo y blanco/gris. Las piezas inca incluyen: morado, negro/rojo, negro/ ante, negro/morado, negro/naranja, negro/ante y morado, rojo/crema y policromo.

Las ollas subglobulares presentan solamente los tratamientos negro/rojo pintado pulido, blanco y negro/rojo pintado pulido, gris pintado pulido y rojo pintado pulido.

\section{Diseño decorativo}

Para el relevamiento del diseño decorativo se consideraron tres aspectos: unidad(es) de diseño, arreglo espacial y estructuración de la(s) unidad(es) de diseño.

La unidad de diseño define al componente menor que puede aislarse en la decoración. En el caso de las vasijas procedentes de la Quebrada de Humahuaca son de dos tipos, unidad de diseño pintada y unidad de diseño incisa, siendo mayoritarias las primeras. La unidad de diseño puede estar formada por un único elemento de diseño (por ejemplo, línea recta, triángulo, espiral), dos o más elementos de diseño que se repiten (por ejemplo, líneas rectas paralelas, incisiones) o la combinación de dos elementos de diseño diferentes (por ejemplo, triángulo con espiral).

Se han definido un total de 76 unidades de diseño pintadas agrupadas en 8 categorías generales (Líneas, Bandas, Triángulos, Círculos, Arriñonados, Espirales, Dameros y Otros) para las piezas locales y 15 para las piezas inca.

Las unidades de diseño incisas comprenden cinco tipos: (A) punteado irregular en los cuellos de las vasijas; (B) hileras paralelas de puntos; (C) puntos formando triángulos con sus vértices para abajo; (D) puntos formando triángulos con sus vértices para abajo delimitados por una línea incisa; (E) bandas imbricadas o corrugadas.

Las ollas subglobulres presentan un total de 20 unidades de diseño pintadas (Tabla 1) del repertorio completo definido para las piezas de la quebrada y los cinco tipos de unidades de diseño incisas (Tabla 2).

El arreglo espacial se refiere al sector de la pieza donde aparecen las unidades de diseño (labio, borde, cara interna, cara externa). 


\section{1, PUCOS}
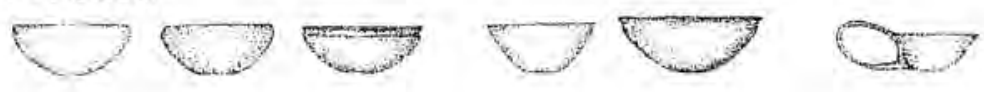

II - OLLAS
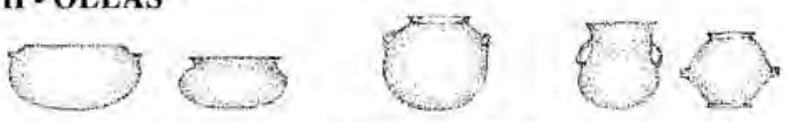<smiles>[As]C1CCCCC1</smiles>

Olla subglobular

\section{III - CANTAROS}

IV - JARRAS
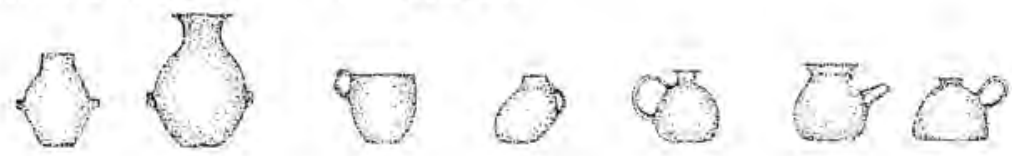

V - VASOS CHATOS

VI - PELIKES
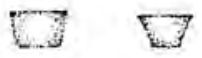

\section{VII - ARIBALIFORMES}<smiles>C1CCCC1</smiles>
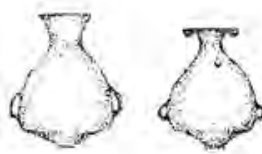

VIII - PLATOS

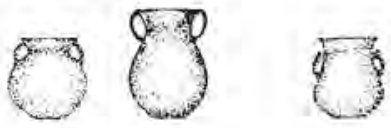

Figura 1: Formas cerámicas de la Quebrada de Humahuaca.

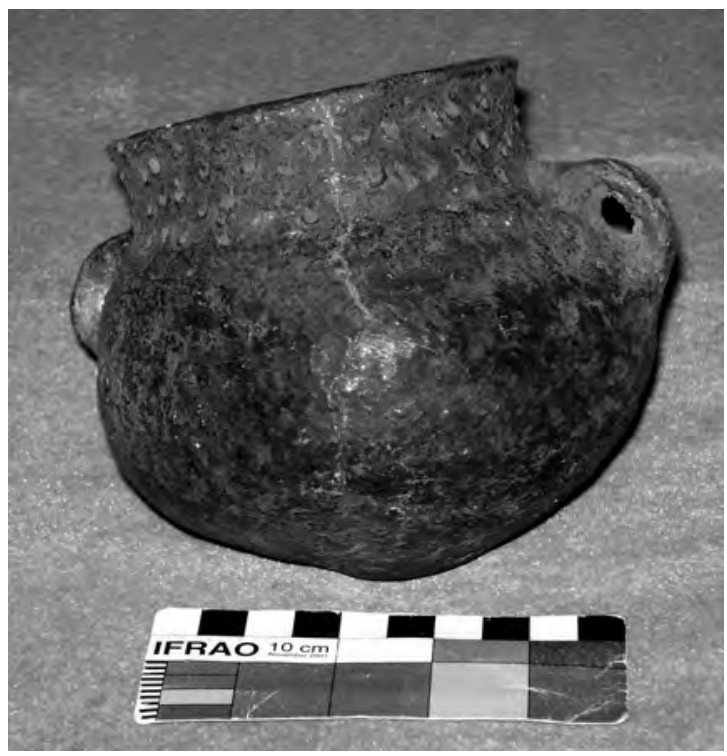

Figura 2: Olla subglobular. 
Tabla 1: Unidades de diseño pintadas presentes en las ollas subglobulares.

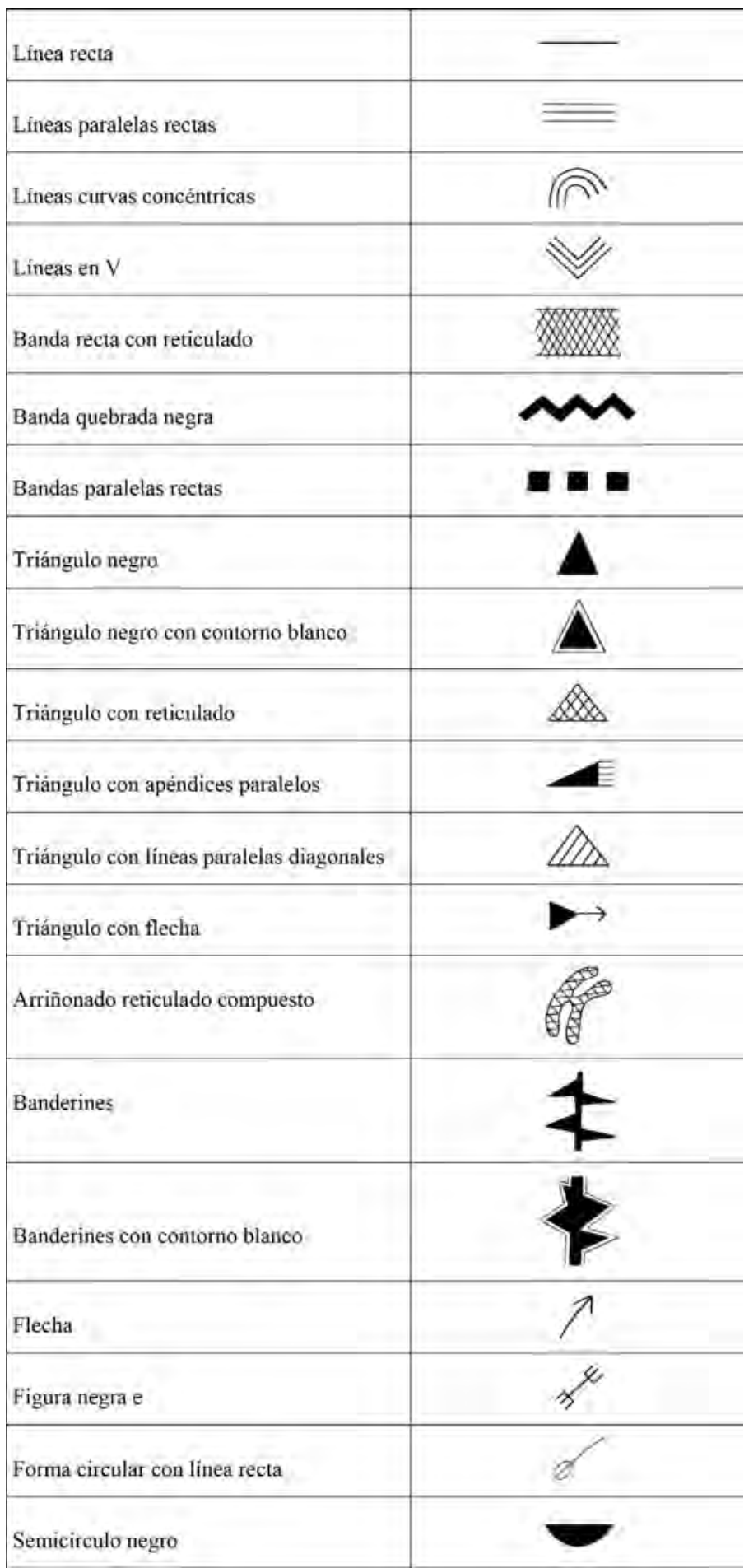


María Andrea Runcio / Estilo y decoración: metodología para el relevamiento de vasijas cerámicas...

Tabla 2: Unidades de diseño incisas presentes en las ollas subglobulares

\begin{tabular}{|c|c|}
\hline Tipo A & Tipo B \\
\hline Tipo C & \\
\hline Tipo D & \\
\hline Tipo E & \\
\hline
\end{tabular}

Tabla 3: Patrones de estructuración presentes en las ollas subglobulares.

Banda vertical


En el caso de las ollas subglobulares corresponde solamente al labio, borde interno y cara externa.

La estructuración define la forma en que se disponen las unidades de diseño en la pieza y puede ser de dos tipos:

- Unidad de diseño aislada (UDA): una única unidad de diseño dispuesta de acuerdo a diferentes patrones.

- Grupo de unidades de diseño (GUD): conjunto de unidades de diseño dispuestas de acuerdo a diferentes patrones.

Los patrones se definen de la siguiente manera: Central, Circular, Tripartito, Cuatripartito, Radial, Lineal, Cobertura total, Curvilíneo, Banda vertical, Banda horizontal, Bandas cruzadas, En serie, Continuo y Disperso.

Las ollas subglobulares sólo presentan los tipos Banda horizontal, Banda vertical, En serie y Disperso (Tabla 3).

Cabe aclarar que nuestra propuesta resulta similar a la de Jerningan (1986) quien plantea un análisis no jerárquico de la decoración, en tanto la definición de "unidad de diseño" entendida como el componente menor que puede aislarse en el diseño sumado al arreglo espacial y la estructuración resulta similar a la noción de "schema" de este autor (ver supra).

Estas variables fueron registradas en una ficha de relevamiento especialmente diseñada. Por último, se elaboró una base de datos en programa Excel que permitió el cruce entre las variables de análisis propuestas, posibilitando sistematizar la información y agilizar el procesamiento de los datos.

\section{ANÁlisis dE LAS OLLAS SUBGLOBULARES}

Se ha estudiado un total de 79 ollas subglobulares.

El tratamiento de superficie predominante en la cara externa es el negro/rojo pintado pulido (32), seguido por el gris pintado pulido (22) y el alisado (18), y en menor frecuencia el rojo pintado pulido (4) y el blanco y negro/rojo pintado pulido (3). En cuanto al borde interno, la mayoría se presenta alisado (61) y en menor medida negro/rojo pintado pulido (17) con un único caso de rojo pintado pulido.

Con respecto al diseño decorativo, 60 ollas se presentan sin unidades de diseño en el labio mientras que 11 presentan unidades de diseño incisas y 8 presentan unidades de diseño pintadas que corresponden a "línea recta".

Para la cara externa, 35 ollas presentan unidades de diseño pintadas y 30 poseen unidades de diseño incisas mientras que 14 ollas no presentan unidades de diseño. Las unidades de diseño pintadas más representadas son la "banda recta con reticulado" y la "línea recta" mientras que entre las unidades de diseños incisas predominan las de tipo A y B (Tabla 4).

En relación al borde interno, la mayoría se presenta sin unidades de diseño (62) mientras que aquellas que las poseen, corresponden a unidades de diseño pintadas siendo predominantes las "bandas paralelas rectas" y las "líneas paralelas rectas" (Tabla 5).

Si correlacionamos el tratamiento de superficie con el diseño decorativo, se observa que aquellas que presentan unidades de diseño pintadas poseen el tratamiento negro/rojo pintado pulido y negro y blanco/rojo pintado pulido y aquellas que presentan unidades de diseño incisas tienen la superficie externa alisada o gris pintada pulida. También se presentan ollas sin unidades de diseño con tratamientos rojo pintado pulido o alisado. 
Tabla 4: Unidades de diseño pintadas e incisas presentes en la cara externa de las ollas subglobulares.

\begin{tabular}{|l|c|}
\hline \multicolumn{1}{|c|}{ Unidades de diseño pintadas } & Total \\
\hline Banda recta con reticulado & 21 \\
\hline Línea recta & 18 \\
\hline Triángulo negro & 4 \\
\hline Arriñonado reticulado compuesto & 3 \\
\hline Banderines con contorno blanco & 3 \\
\hline Triángulo negro con contorno blanco & 3 \\
\hline Triángulo con reticulado & 2 \\
\hline Banderines & 2 \\
\hline Tríngulo con apéndices paralelos & 2 \\
\hline Flecha & 1 \\
\hline Triángulo con líneas paralelas diagonales & 1 \\
\hline Figura negra e & 1 \\
\hline Forma circular con linea recta & 1 \\
\hline Triángulo con flecha & 1 \\
\hline & \\
\hline Unidades de diseño incisas & 13 \\
\hline B & 8 \\
\hline D & 4 \\
\hline E & 3 \\
\hline C & 2 \\
\hline
\end{tabular}

Tabla 5: Unidades de diseño pintadas presentes en el borde interno de las ollas subglobulares

\begin{tabular}{|l|c|}
\hline \multicolumn{1}{|c|}{ Unidades de diseño pintadas } & Total \\
\hline Bandas paralelas rectas & 6 \\
\hline Líneas paralelas rectas & 5 \\
\hline Semicírculo negro & 2 \\
\hline Lineas curvas concéntricas & 1 \\
\hline Banda quebrada negra & 1 \\
\hline Líneas en V & 1 \\
\hline Triángulo negro con contorno blanco & 1 \\
\hline
\end{tabular}

Tabla 6: Patrones de estructuración presentes en la cara externa y borde interno de las ollas subglobulares.

\begin{tabular}{|l|c|c|}
\hline \multicolumn{1}{|c|}{ Estructuración } & Cara externa & Borde interno \\
\hline UDA Disperso & 27 & - \\
\hline UDA Banda horizontal & 12 & - \\
\hline GUD Banda horizontal & 10 & - \\
\hline GUD Banda vertical & 7 & - \\
\hline UDA En serie & 5 & 16 \\
\hline GUD En serie & 2 & 1 \\
\hline UDA Banda vertical & 2 & - \\
\hline Sin estructuración & 14 & 62 \\
\hline Total & 79 & 79 \\
\hline
\end{tabular}


Con respecto a la estructuración, para el labio se presenta la unidad de diseño aislada con patrón continuo y la unidad de diseño aislada con patrón en serie, en correspondencia con las unidades de diseño presentes en el mismo ("línea recta" e "incisiones"). Para la cara externa la más representada es la unidad de diseño aislada con patrón disperso y para el borde interno, la unidad de diseño aislada con patrón en serie (Tabla 6).

Finalmente, se ha analizado la combinación de unidades de diseño pintadas en la cara externa (que se presentan sobre tratamientos, negro/rojo pintado pulido y negro y blanco/rojo pintado pulido) y su estructuración a través de los diferentes patrones, lo que ha permitido definir un conjunto de Grupos (Tabla 7). Es decir, los Grupos comprenden todas las ollas que presentan la misma combinación de unidades de diseño pintadas y patrón de estructuración. Cabe destacar, sin embargo, que algunas ollas poseen diseños pintados o combinaciones de los mismos y patrones de estructuración que se presentan una sola vez en el total de ollas estudiadas y fueron incluidas en el Grupo 8.

Por otro lado, al realizar un análisis más detallado de cada una de las ollas que forman parte de los distintos Grupos se ha podido determinar el agregado de lo que podría definirse como "detalles" que particularizan algunas ollas dentro del "grupo general" en el que fueron clasificadas.

Estos “detalles" incluyen (Tabla 8):

Grupo 1: A- La "banda recta con reticulado" está bordeada por líneas blancas. B- Se agrega "línea recta". C- Se agrega "figura negra e" en ambas asas.

Grupo 2: Se presenta sin "línea recta".

Grupo 3: Se reemplaza el "triángulo negro" por "triángulo con reticulado".

Grupo 7: A- Se incorpora "triángulo negro" y "forma circular con línea recta". B- Se agrega "flecha”. C- Se agrega "triángulo con flecha".

\section{COMENTARIO FINAL}

La Quebrada de Humahuaca presenta una unidad estilística a nivel regional que se remonta al período Medio (700-900 d.c.) donde comienza a configurarse una tradición alfarera que continúa, con algunas modificaciones en la forma y decoración de las vasijas, durante los períodos Tardío e Inca (900-1536 d.C.). Puede decirse que hay un lenguaje plástico común -manifestado en criterios compartidos sobre la producción de ciertas formas, el uso de determinados tratamientos de superficie y unidades de diseño y la manera de combinarlas y estructurarlas en distintos sectores de las piezasque otorga unidad estilística al conjunto de vasijas que identifica a las sociedades de la Quebrada de Humahuaca.

Las ollas subglobulares analizadas en este trabajo se integran a esta tradición estilística regional compartiendo acabados de superficie, diseños pintados e incisos y patrones de estructuración con las otras formas cerámicas presentes en la Quebrada de Humahuaca.

Sin embargo, cuando se profundizó en el análisis de las mismas comenzaron a surgir particularidades manifestadas en pequeños "detalles" que individualizan a algunas ollas dentro del Grupo en el

2.- Considero como "detalle" el agregado de diseños pintados que se combinan con aquellos que caracterizan a cada uno de los Grupos, la ausencia de uno de los diseños pintados característico de cada Grupo o alguna pequeña modificación en la ubicación de los diseños pero sin que se altere el patrón de estructuración que define al Grupo. 
Tabla 7: Grupos correspondientes a las ollas subglobulares.

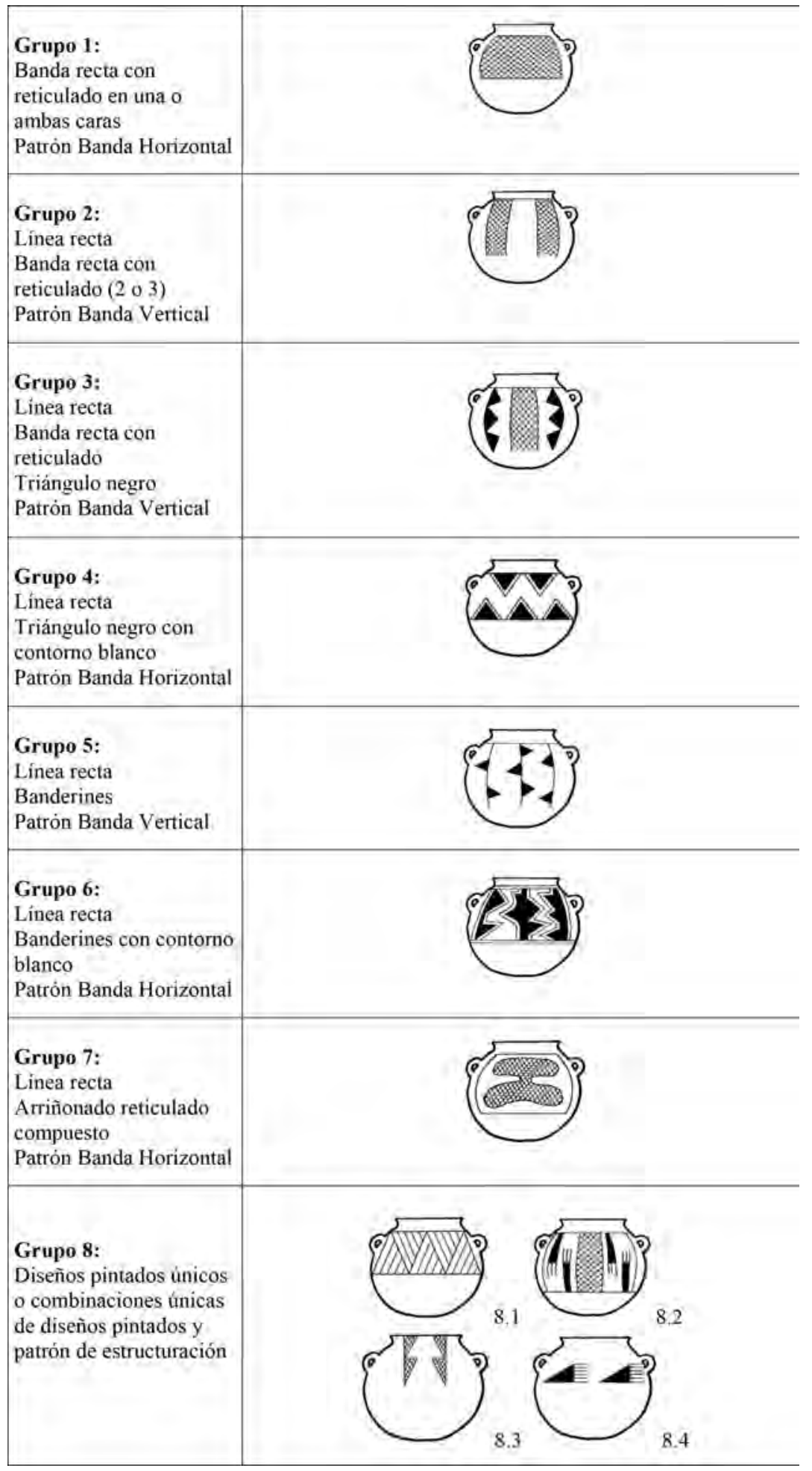


Tabla 8: "Detalles" correspondientes a las ollas subglobulares.

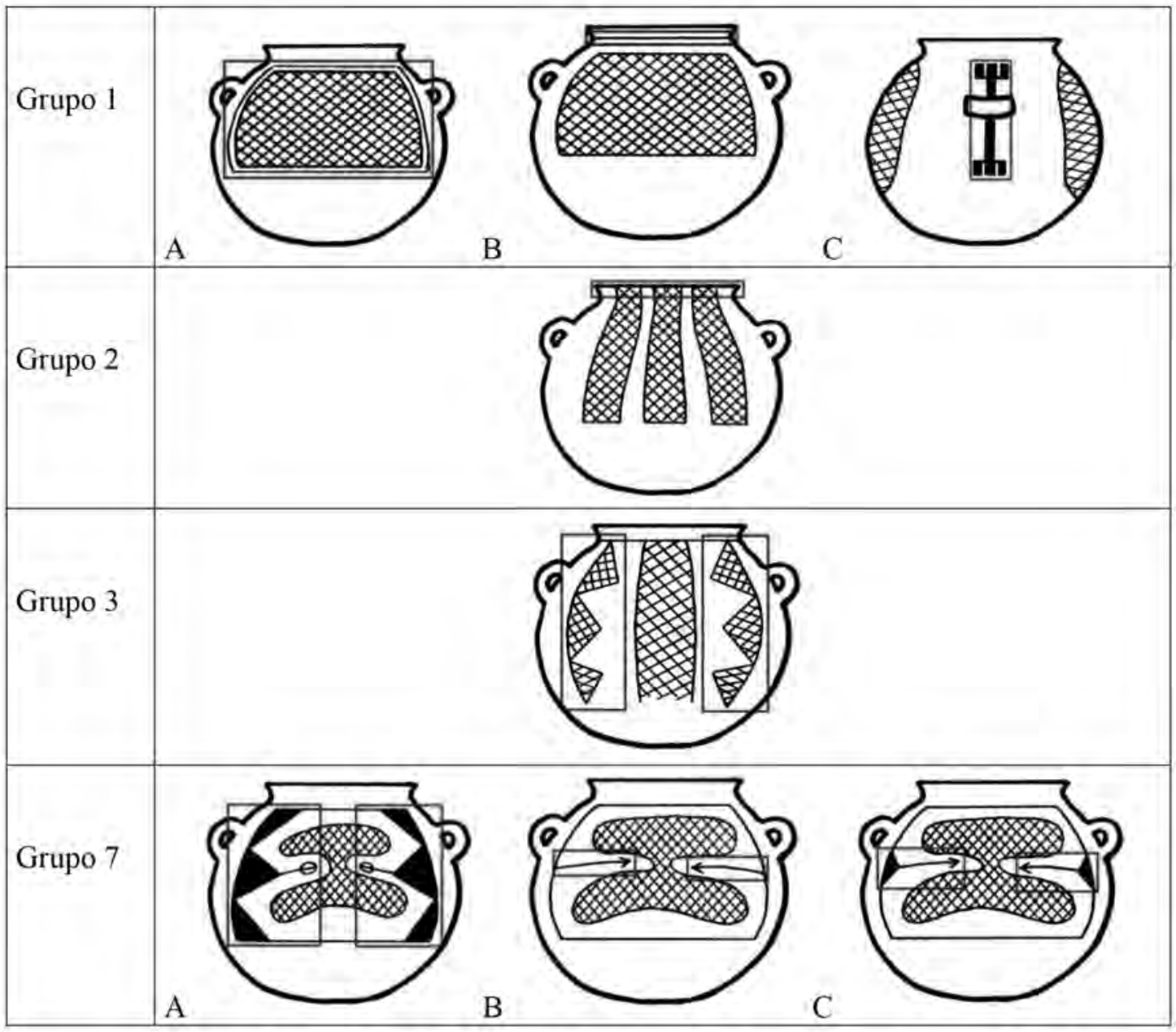


cual fueron clasificadas y otras que poseen diseños pintados o combinaciones de los mismos y patrones de estructuración que se presentan representados una sola vez (Tabla 7 y 8 ).

Coincidimos con Wells (1998: 241) en que la mayoría de los enfoques tradicionales en arqueología han sido fuertemente normativos enfatizando el desarrollo de secuencias tipológicas y las entidades que no se ajustaban a las generalizaciones tendían a ser ignoradas o descartadas como excepciones que no requieren explicación. El resultado de este enfoque ha sido el des-énfasis de la individualidad y de la variabilidad en los patrones de la evidencia arqueológica.

La Quebrada de Humahuaca no es una excepción y las secuencias estilísticas construidas tempranamente han sido cuestionadas (Palma 1996; Nielsen 1997; Rivolta 1997). En consideración a ello, se dejó de lado la clasificación de las vasijas de acuerdo a los estilos definidos para el área y se desarrolló una metodología que partió del análisis de variables independientes y su posterior correlación, permitiendo no constreñir la clasificación de la decoración de las vasijas en categorías estrechas. A partir de la misma, entonces, fue posible dar cuenta no solo de la coherencia implícita en todo estilo sino también de la variación inherente al mismo verificada en elementos únicos, que en nuestro caso se manifiestan a través de algunas piezas con el agregado de "detalles" y otras con diseños pintados o combinaciones de los mismos que aparecen representados una sola vez en el total de vasijas decoradas.

\section{Agradecimientos}

A Gabriela Amirati, Silvia Manuale, Victoria Coll y Alejandra Reynoso (Depósito de Arqueología, Museo Etnográfico), Jorge Kraideber y Gabriel Alarcón (Museo de La Plata) y Armando Mendoza (Museo E. Casanova) por haberme facilitado el acceso a las colecciones y a Irene Lantos por su orientación para la construcción de la base de datos.

\section{BiBLIOGRAFÍA}

\section{DOUGLASS, Amy y Owen LINDAUER}

1988 Hierarchical and nonhierarchical approaches to ceramic design analysis: a response to Jerningan. American Antiquity, 53(3): 620-626.

HODDER, Ian

1985 Postprocessual Archaeology. Advances in Archaeological Method and Theory, 8: 1-26.

\section{JERNIGAN, E. Wesley}

1986 A nonhierarchical approach to ceramic decoration analysis: a southwestern example. American Antiquity, 51(1): 3-20.

\section{LONGACRE, William}

1968 Sociological implications of the ceramic analysis. Chapters in the prehistory of eastern Arizona II, P. Martin, J. Rinaldo, W. Longacre, L. Freeman, J. Brown, R. Hevly y M. Cooley (eds.), pp. 155-170. Fieldiana Anthropology 55, Chicago.

\section{LÓPEZ, Mariel}

2000-2002 Técnicas de acabado de superficie de la cerámica arqueológica: indicadores macro y microscópicos. Una revisión sobre las técnicas de estudio más habituales. Cuadernos del Instituto Nacional de Antropología, 19:347-364. Instituto Nacional de Antropología, Buenos Aires. 
LÓPEZ, Mariel

2004 Tecnología cerámica en La Huerta, Quebrada de Humahuaca, Provincia de Jujuy. Tesis Doctoral. Facultad de Filosofía y Letras, Universidad de Buenos Aires.

NIELSEN, Axel

1997 Tiempo y cultura material en la Quebrada de Humahuaca, 700-1650 d.C. Instituto Interdisciplinario Tilcara, Facultad de Filosofía y Letras, Universidad de Buenos Aires.

PALMA, Jorge

1996 Estructuras de descarte en un poblado prehispánico de la Quebrada de Humahuaca. Arqueología. Revista de la Sección Arqueología. Instituto de Ciencias Antropológicas, 6: 4767. Facultad de Filosofía y Letras, Universidad de Buenos Aires.

PALMA, Jorge

1998 Curacas y Señores. Una visión de la sociedad política prehispánica en la Quebrada de Humahuaca. Instituto Interdisciplinario Tilcara, Facultad de Filosofía y Letras, Universidad de Buenos Aires.

RICE, Prudence

1987 Pottery analysis: a sourcebook. Chicago University Press, Chicago.

RIVOLTA, María Clara

1997 Revisión crítica de la obra de Bennett y colaboradores sobre la definición y asignación cronológica de algunos estilos cerámicos de la Quebrada de Humahuaca. Avances en Arqueología, 3: 131-145. Instituto Interdisciplinario Tilcara, Facultad de Filosofía y Letras, Universidad de Buenos Aires.

ROCCHIETTI, Ana María

1991 Estilo y diferencia: un ensayo en área espacial restringida. El arte rupestre en la arqueología contemporánea, M. M. Podestá, M. I. Hernández Llosas y S. Renard de Coquet (eds.), pp. 2530, Salón Gráfico Integral SRL, Buenos Aires.

ROCCHIETTI, Ana María

1995 El arte rupestre del sitio El Ojito (Dpto. de Río Cuarto, Provincia de Córdoba, República Argentina). Revista de la Universidad Nacional de Río Cuarto, 15 (1-2): 81-93. Universidad Nacional de Río Cuarto.

\section{ROCCHIETTI, Ana María}

2002 Arte rupestre en ambiente granítico de la sierra de Comechingones: formación arqueológica y marco teórico. Actas del XIII Congreso Nacional de Arqueología Argentina, Tomo II: 65-74. Córdoba.

\section{ROCCHIETTI, Ana María}

2008 Una propuesta de conceptos para abordar el arte rupestre. Trabajo presentado al III Simposio Nacional de Arte Rupestre. 29 de octubre al 2 de noviembre de 2008. Huaraz, Perú. 
María Andrea Runcio / Estilo y decoración: metodología para el relevamiento de vasijas cerámicas...

\section{RUNCIO, María Andrea}

2010 Estilos e identidades: producción y consumo de vasijas cerámicas en la Quebrada de Humahuaca durante los períodos Tardío e Inca (900-1536 d.C.). Tesis Doctoral. Facultad de Filosofía y Letras, Universidad de Buenos Aires.

SACKETT, James

1977 The meaning of style in archaeology: a general model. American Antiquity, 42: 362-380.

SHANKS, Michael y Christopher TILLEY

1992 Re-constructing archaeology. Theory and practice. Routledge, London.

WELLS, Peter

1998 Identity and material culture in later prehistory of Central Europe. Journal of Archaeological Research, 6(3): 239-298.

WIESSNER, Polly

1983 Style and social information in the Kalahari San projectile points. American Antiquity, 48(2): 253-276.

WOBST, Martin

1977 Stylistic behavior and information exchange. Papers for the Director: research essays in honor of J. Griffin. C. Cleland (ed.). Museum of Anthropology, Anthropological Papers № 61: 317-342. University of Michigan, Ann Arbor. 
